\title{
Conductive Carbon Coatings for Electrode Materials
}

Marca M. Doeff, ${ }^{a}{ }^{*}$ Robert Kostecki, ${ }^{b}$ James Wilcox, ${ }^{\text {a }}$ and Grace Lau ${ }^{\text {a }}$

a) Materials Sciences Division

and

b) Environmental Energy Technologies Division

Lawrence Berkeley National Laboratory

1 Cyclotron Road

Berkeley, CA 94720

\section{Acknowledgment}

This work was supported by the Assistant Secretary for Energy Efficiency and Renewable Energy, Office of FreedomCAR and Vehicle Technologies of the U.S. Department of Energy under contract no. DE-AC02-05CH11231.

* e-mail: mmdoeff@lbl.gov tel: (510 ) 486-5821 
The Pitch

A simple method for optimizing the carbon coatings on non-conductive battery cathode material powders has been developed at Lawrence Berkeley National Laboratory. The enhancement of the electronic conductivity of carbon coating enables minimization of the amount of carbon in the composites, allowing improvements in battery rate capability without compromising energy density.

The invention is applicable to $\mathrm{LiFePO}_{4}$ and other cathode materials used in lithium ion or lithium metal batteries for high power applications such as power tools and hybrid or plug-in hybrid electric vehicles. The market for lithium ion batteries in consumer applications is currently $\$ 5$ billion/year. Additionally, lithium ion battery sales for vehicular applications are projected to capture 5\% of the hybrid and electric vehicle market by 2010 , and $36 \%$ by 2015 (http://www.greencarcongress.com).

$\mathrm{LiFePO}_{4}$ suffers from low intrinsic rate capability, which has been ascribed to the low electronic conductivity $\left(10^{-9} \mathrm{~S} \mathrm{~cm}^{-1}\right)$. One of the most promising approaches to overcome this problem is the addition of conductive carbon. Co-synthesis methods are generally the most practical route for carbon coating particles. At the relatively low temperatures $\left(<800^{\circ} \mathrm{C}\right)$ required to make $\mathrm{LiFePO}_{4}$, however, only poorly conductive disordered carbons are produced from organic precursors. Thus, the carbon content has to be high to produce the desired enhancement in rate capability, which decreases the cathode energy density.

\section{The Technology}

When certain readily decomposed organic compounds and graphitization catalysts are chosen as precursors, conductive carbons with more graphitic structures are produced at temperatures compatible with synthesis of $\mathrm{LiFePO}_{4}$ (patent pending). Small amounts of the organic compounds and the inorganic or organometallic catalysts are initially added to the precursor mix prior to calcination. The optimized process results in a phase-pure $\mathrm{LiFePO}_{4}$ product consisting of small particles homogeneously coated with a less than 10 nm thick graphitic carbon layer (Figure 1), which can withstand prolonged cycling and high rate discharges. Carbon coatings on $\mathrm{LiFePO}_{4}$ produced by LBNL's new technology result in an enhancement of the composite conductivity of as much as six orders of 
magnitude. The key to these features is the less disordered and more graphitic structure of the carbon arising from the additives used during co-synthesis, as determined by Raman spectroscopy (Figure 1 inset). This results in higher composite conductivity and are closely correlated with the improved rate capabilities in lithium cells at low carbon contents $(<2$ wt. \%). For example, a one order magnitude increase in composite conductivity arising from a $25 \%$ higher $\mathrm{sp}^{2} / \mathrm{sp}^{3}$ ratio results in up to $45 \%$ better electrochemical utilization in lithium batteries when comparing coated $\mathrm{LiFePO}_{4}$ materials having the same carbon contents. The proposed additives and methodologies are low-cost and can easily be integrated into existing manufacturing processes.

\section{Opportunities}

The researchers and LBNL are seeking licensees for their conductive coating technologies and academic and industrial partners for collaborative R\&D.

\section{Sources}

Marca M. Doeff, M/S 62R0100, Lawrence Berkeley National Laboratory, Berkeley, CA 94720, USA; tel. 510 486-5821 and e-mail mmdoeff@lbl.gov and Robert Kostecki, M/S 70-108B, Lawrence Berkeley National Laboratory, Berkeley, CA 94720, USA; tel (510) 486-6002 and e-mail R_Kostecki@lbl.gov. For licensing: Michael Mueller, LBNL Technology Transfer Department, M/S 90R1070, Lawrence Berkeley National Laboratory, Berkeley, CA 94720, USA; tel. $510 \quad 486-4303$ and e-mail MMMueller@lbl.gov.

\section{Figure Captions}

Figure 1. SEM picture of a coated $\mathrm{LiFePO}_{4}$ particle, and a typical Raman spectrum of the carbon coating. Deconvoluted peaks correspond to $\mathrm{sp}^{3}$-, and disordered (D) and graphene (G) bands of sp $^{2}$-type carbon. 


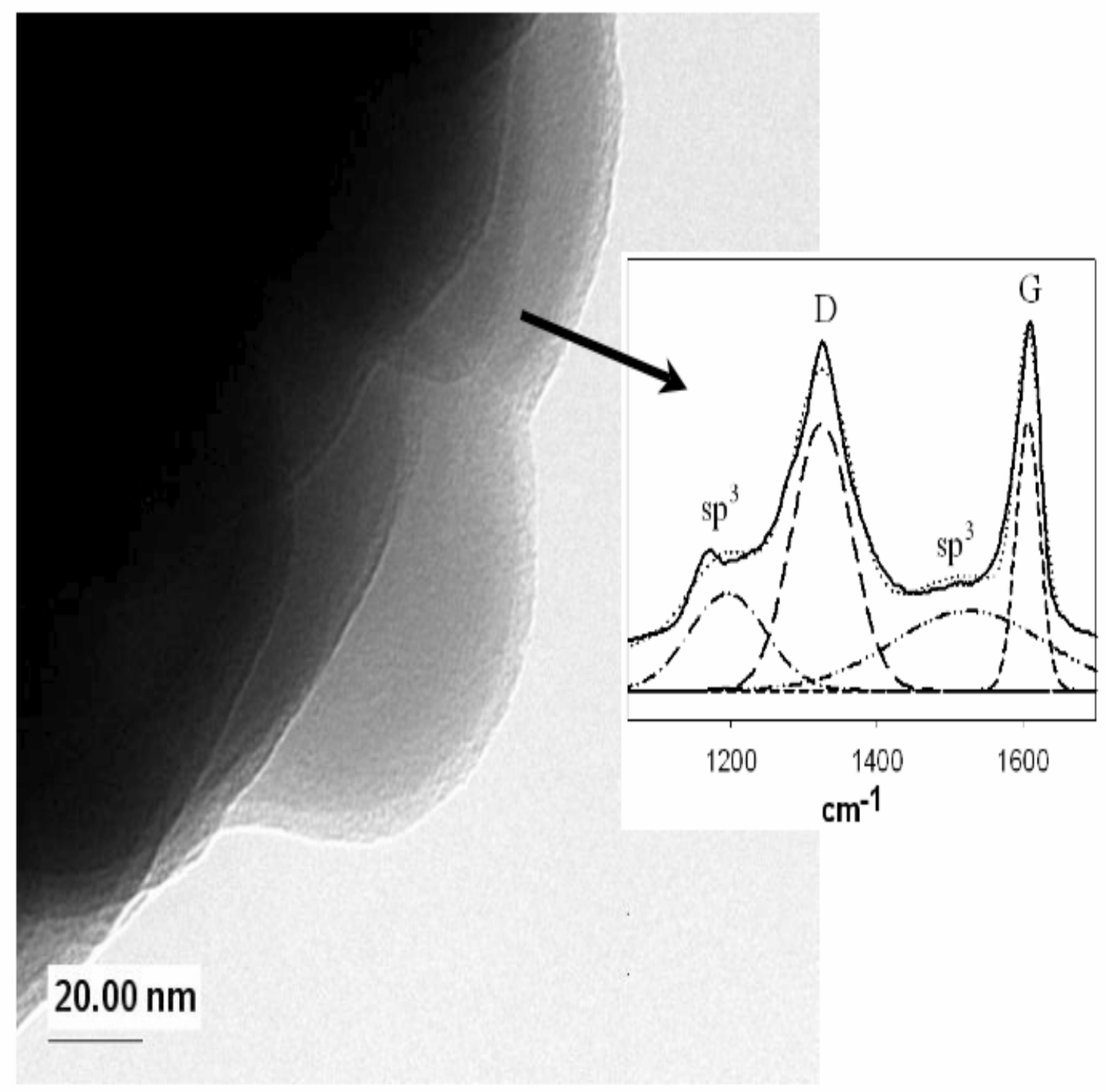

Figure 1 